\title{
An Integrated Power Grid Equipment Operation and Maintenance Solution Based on Big Data, Cloud Computing, the Internet of Things and Mobile Internet
}

\author{
Liang Dong ${ }^{1}$, Su Yirong ${ }^{1,2, ~ *, ~ L i u ~ Z h i w e i ~}{ }^{2}$, Liu Shen ${ }^{1}$ \\ ${ }^{1}$ State Grid Jibei Electric Power Company, Beijing, China \\ ${ }^{2}$ NARI Group Corporation, Nanjing, China
}

Email address:

liang.dong@jibei.sgcc.com.cn (Liang Dong), suyirong@sgepri.sgcc.com.cn (Su Yirong), liuzhiwei @ sgepri.sgcc.com.cn (Liu Zhiwei), liu.shen@jibei.sgcc.com (Liu Shen)

${ }^{*}$ Corresponding author

\section{To cite this article:}

Liang Dong, Su Yirong, Liu Zhiwei, Liu Shen. An Integrated Power Grid Equipment Operation and Maintenance Solution Based on Big Data, Cloud Computing, the Internet of Things and Mobile Internet. Automation, Control and Intelligent Systems. Vol. 5, No. 5, 2017 , pp. 67-72. doi: $10.11648 /$ j.acis.20170505.12

Received: September 1, 2017; Accepted: October 10, 2017; Published: October 31, 2017

\begin{abstract}
The traditional operation and maintenance methods cannot meet the new demands of current grid operation and maintenance as it grows in both scale and complexity. Based on the analysis of the shortcomings of the existing methods, an integrated solution based on big data, cloud computing, the Internet of Things and mobile Internet is proposed in this paper. Internet of Things technologies such as RFID and wireless transmission are adopted to acquire and sense information of field equipment. Big data and cloud computing technologies are used to standardize field work management and supervise standard field work operation. Besides, a data exchange channel is built between maintenance site and control center via intelligent operation and maintenance terminals to enable data upload and interaction between maintenance site and control center.
\end{abstract}

Keywords: Integrated Operation and Maintenance, The Internet of Things, Big Data, Cloud Computing, Intelligent Operation and Maintenance

\section{Research Background}

The operation and maintenance (O\&M) of the grid equipment is critically important to guarantee the stability and reliable of the power grid. It is also an important foundation for the upgrading of customer services of the power utilities. With the increase of power grid scale and the higher requirements put on customer services, the task of grid O\&M becomes harder gradually. Limited by the O\&M capacity, the human-based traditional mode of grid O\&M has been unable to meet the increasingly complex requirements. In traditional mode, data of different sectors are isolated and operators cannot manage grid in real-time as there is no way to effectively analyze and diagnose grid status. The traditional mode has the following drawbacks: (1) The inspection and maintenance work scope is limited and the maintenance work is cost-inefficient and lacks efficiency and controllability. (2) Mainly based on human, the maintenance work has higher possibility of randomness and mistakes. In case of unexperienced personnel, his/her operation would be unstandardized. The operators cannot guide field work in his/her office. (3) Equipment accounts are recorded manually on paper. Different staff would have different understanding of the recording requirements and make mistakes during recording. Therefore, the data are lack of standardization. Besides, troubleshooting time would be extended due to different data forms of different manufacturers. (4) There are many blind sites during maintenance work. The work process is not standardized and lack of control and management. There are few field-work-oriented applications. To summarize, the traditional mode lacks standard management means and information-based procedure and does not suited to the current requirements of smart grid. There is a large gap between the traditional mode and modern mode in terms of management and operation means. The traditional mode cannot support power supply reliability and high power 
quality any more.

The integrated O\&M solution based on "big data, cloud computing, the Internet of Things and mobile Internet" enables interoperability and interaction through adopting Internet of things and mobile Internet technology. It also develops practical applications using big data and cloud computing technology. It can facilitate maintenance work via providing information, standards and regulations.

Big data, cloud computing, the Internet of Things and mobile Internet technologies are centered on big data, based on cloud computing and Internet of Things and uses mobile Internet as an interactive method. Big data and cloud computing exploit the potential value of resources and dynamically allocate resources through data analysis and information sharing. Internet of things and mobile Internet enables data exchange and information interaction between people and equipment. At present, these technologies have been successfully applied in fields such as safety supervision, logistics, smart city, industrial control, medical and other fields. In smart city field, big data, cloud computing, the Internet of Things and mobile Internet technologies have been used in our lives as basic infrastructure. Compared to other technologies, they are more intelligent and can provide better user experience They enables automated observability of activities and provides cost-effective services through real-time high-resolution information capture and prediction. They can analyze performance information of various devices in real-time and improves operational efficiency. In order to promote the application of big data, cloud computing, the Internet of Things and mobile Internet technologies in power grid and the scientific development of communication technology and IT industry, the State Grid has adopted a series of measures to enhance the application of "big data, cloud computing, the Internet of Things and mobile Internet" in grid. In September 2015 State Grid Corporation released Action Plan of Using New Information and Communication Technologies to Promote Smart Grid and Support the Innovative Development " a Strong and Three Excellent "Modern Company. In November, the same year, the action plan was encompassed in the "13th Five-Year Plan" and special plans of power grid, information, technology, construction, dispatching, O\&M, marketing and industry sectors. In July 2016 during State Grid's annual meeting in the middle of the year, Shu Yin Biao, the chairman once again stressed the importance of "big data, cloud computing, the Internet of Things and mobile Internet" technologies for power grid development, and advocated to "accelerate the construction of integrated 'national grid cloud platform. Appraising Meeting of Key Technology Research and Demonstration Project of Grid Big Data Platform project, organized by China Industry Software Association, was held in Beijing on March 11, 2016. The project is an active application of big data, cloud computing, the Internet of Things and mobile Internet technologies by State Grid. The platform has been applied in 10 utilities, such as Jiangsu, Shanghai, Zhejiang power utilities and State Grid Service Center. The platform realizes typical applications in 10 types of scenarios such as power load forecasting, lean-oriented management of distribution grid troubleshooting, grid equipment status monitoring etc. The project achieved significant economic and social benefits. The management means of State Grid has been brought into a new stage through the promotion of the integration of Internet of things and marketing services, business expansion, O\&M, asset management, and other core businesses.

Big data, cloud computing, the Internet of Things and mobile Internet technologies is mainly adopted in O\&M sector in terms of their applications in power grid. These technologies are important means to promote O\&M intelligence. In order to achieve intelligent O\&M, we should take the initiative to comply with the development Internet of things and smart grid, promote development of intelligent O\&M, the integration between modern information and communication technology and Intelligent technology and power grid O\&M, and technologies such as real-time information acquisition, discrete data acquisition and transmission, mass maintenance data mining. Intelligent O\&M can achieve the optimal allocation and efficient operation inspection resources. This paper proposes an integrated power grid equipment O\&M solution based on big data, cloud computing, the Internet of Things and mobile Internet. The solution combines intelligent technologies and O\&M via the use of relation among equipment and modules. It makes up for the drawbacks of traditional O\&M mode and provides technical support means for O\&M.

\section{O\&M Solution}

\subsection{Solution Objective}

As big data, cloud computing, the Internet of Things and mobile Internet technologies are promoting smart grid construction and State Grid, a company with one strength and three excellencies are advocating innovative and Internet + applications, developing new O\&M technologies becomes extremely critical. It is also one of the directions of smart grid development. The integrated O\&M solution aims to solve the problems of the traditional mode and provide O\&M with information support. It acquires information in standard process and manner, which changes manual data recording methods and avoids non-standard data and errors. Thus, efficiency and quality of O\&M work is improved and the security and reliability of system operation is ensured.

\subsection{Technical Solution}

The integrated O\&M solution based on big data, cloud computing, the Internet of Things and mobile Internet is composed of a field work platform, a mobile work platform and a central supporting system. Field work platform is a platform for field work based on intelligent sensing and mobile Internet technologies. It realizes comprehensive awareness of power equipment and equipment management via RFID, wireless transmission and other Internet of things technologies. The platform realizes the interconnection 
between field works and central system via intelligent mobile terminal units. The central system provides data analysis functions and manages field work in a standard manner. The architecture of the solution is shown in Figure 1.

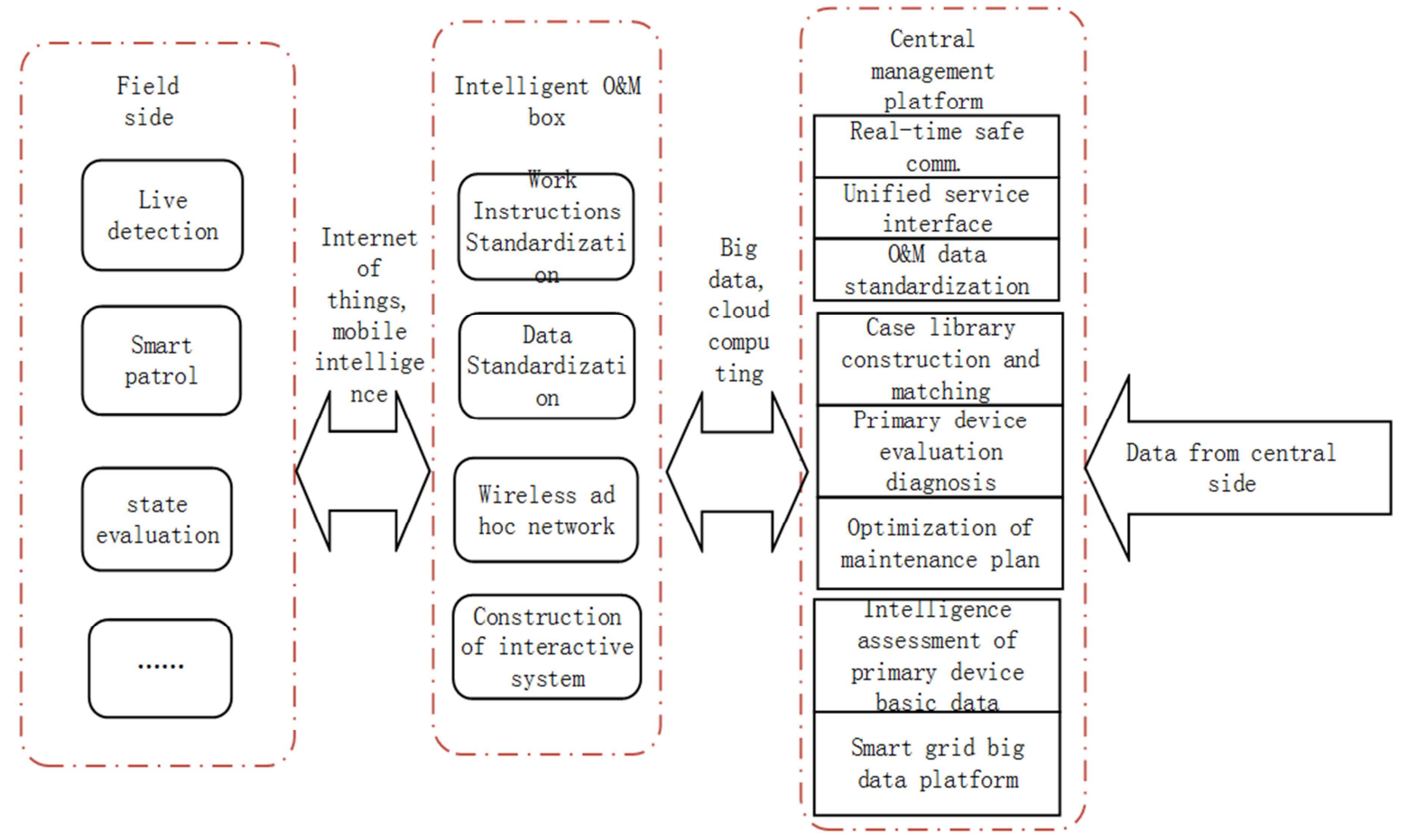

Figure 1. The structure of integrated operation and maintenance solution based on " big data, cloud computing, the Internet of Things and mobile Internet".

(1) Field work platform. The platform dynamic manages information of on-site devices via RFID and other intelligent sensing technology. There is a QR code or electronic tag on each device which is used to connect with mobile work platform. Field work platform can enquire equipment information, historical records and data access service address etc. and conduct live detection, offline test data entry, etc.

(2) Mobile work platform. Various data are standardized in format via intelligent mobile terminal units and through standard data conversion interface. Workflow can be expressed in a standardized and unified format and be uploaded at one-click. Thus, manual data entry will be reduced greatly. The intelligent mobile terminal unit is also connecting platform between field work side and central system side, serving as a platform for on-site assessment and a transit agent. It has access to the cloud-based resources of the central system and can guide field work. The intelligent mobile terminal unit has built-in equipment multidimensional data and state analysis model to provide field-work-related data analysis capabilities. It supports non-network work mode. Communication protocols of more than ten types of live detection equipment and most of the power failure detection equipment is built in the terminal unit. $80 \%$ of instruments can be accessed. The terminal unit complies with State Grid information security system and is with high reliability and portability.

(3) Central system. Central system adopts distributed architecture and can be seamlessly integrated in PMS, intelligent $0 \& \mathrm{M}$ control system etc. The analysis algorithm can be customized through the micro-application of big data technology. Real-time data verification and error correction can be conducted through historical data comparison and real-time evaluation of equipment status can be done through multi-dimensional data analysis.

The field workflow is as the following. O\&M personnel arrives at site with an intelligent mobile terminal unit. After the terminal is turned on, a wireless ad hoc network is automatically generated and the terminal is initialized. The terminal downloads the relevant data and work flow from central system. During the O\&M work process, the personal needs to strictly follow steps presented on the mobile terminal. The field work data can be exchanged with the central side data via intelligent terminal so that on-site worker can use the data resources of control center and the central side can control field work. This mechanism guarantees the quality of field work.

\section{Function Features}

\subsection{Intelligent Terminal Unit}

The intelligent terminal unit is connection between the field side and the control system side. It can automatically generate wireless ad hoc network. The field instrument and the mobile control terminal (such as PAD and mobile phone) can be connected to it, and then be connected to the central side through private network or GPRS.

The intelligent terminal unit has can be divided into two layers from the perspective of functions, i.e. application layer and core layer. The core layer connects with the application 
layer via wireless transmission module such as wifi, 3G / 4G and Bluetooth to, as shown in Figure 2.

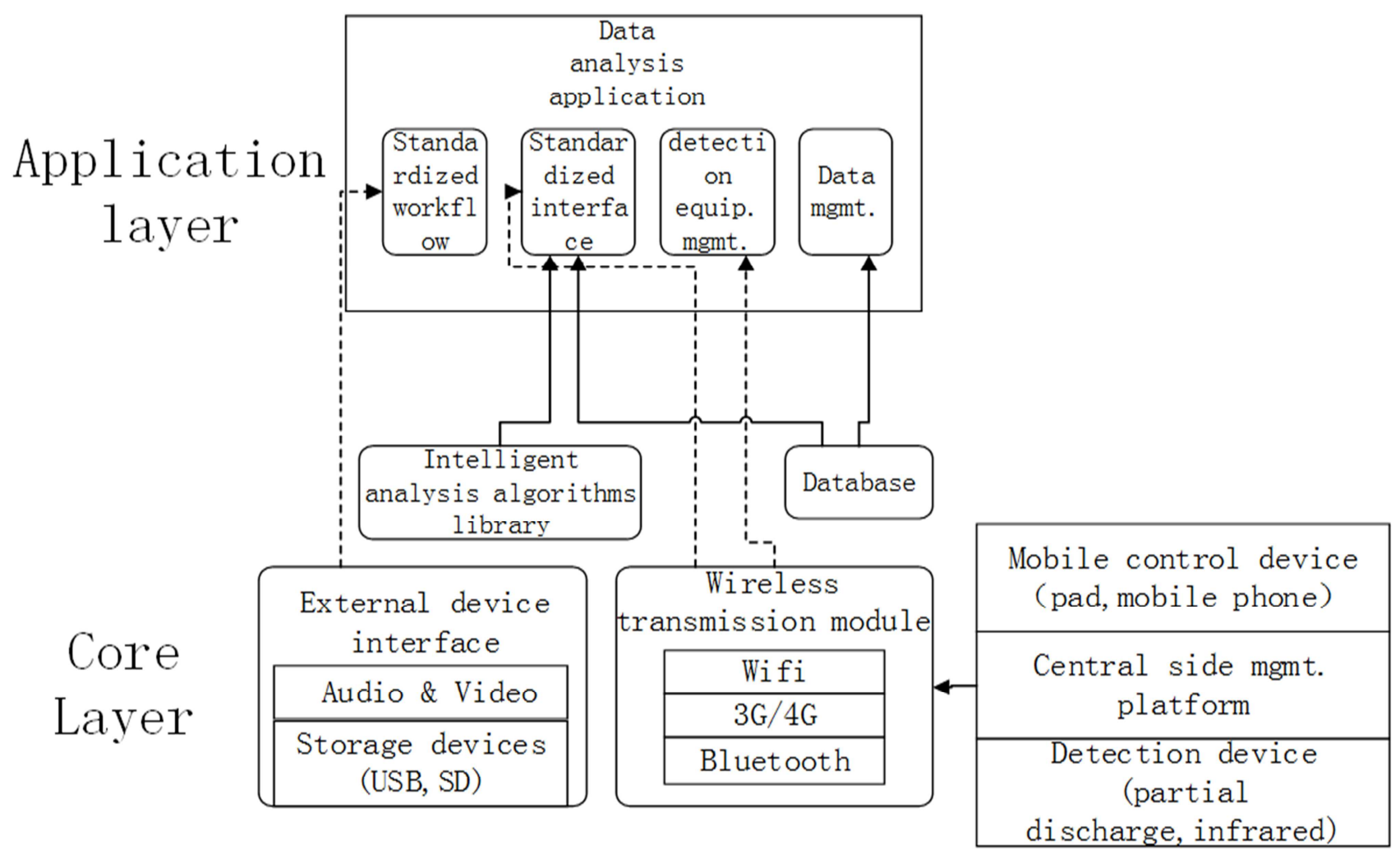

Figure 2. Functions of intelligent operation and maintenance terminals.

The application layer establishes data analysis applications through embedded database and intelligent analysis algorithm library, realizes the standardization of the workflow through normalized data interface and conducts equipment detection management and data management. Standardized workflow function of the application layer is realized by configuring audio and video and external device interfaces such as USB, SD card etc. The standardization of field data and workflow can be interpreted as the following.1) Communication protocols of mainstream test equipment are supported and data conversion interfaces are provided to ensure the unification and standardization of data from the same test projects. 2) Field O\&M guidelines and field work manual are presented in a knowledge base. Field work is broken down into steps and O\&M personnel follows the steps and interactively fill blanks on terminals. This is to ensure the standardization of O\&M work. 3) Video and audio interface is provided in order to record key steps of field work in video or audio.

Data and algorithm model of the intelligent terminal unit are periodically updated from control system to support data analysis function. Customized services can be provided for special substations and O\&M requirements. Data and similar cases can be remotely called from the central side. It is also possible to call calculations and analysis resources of the central side for advanced analysis. The mobile terminal supports video component access, and in special cases experts from the central side can provide guidance remotely.

\subsection{Information Security Protection}

Four layers of protection are used for information security: (1) security terminal layer: built-in terminal encryption chip, issued by the PKI service system for digital certificate issuance.(2) Secure channel layer: Provides secure network access between terminal and access platform, including wired private line, wireless line APN etc. (3) Access system layer: Access system layer is the core component of the system, composed by security access gateway system, identity authentication system, security data filtering system and central monitoring and management system.(4) Business service layer: mainly including the background service system of application systems, front-end servers etc. Operating system protection, password protection, access authentication protection etc. are adopted.

\subsection{Central Supporting System}

The central system includes a prompt module, a data analysis module and a case query module, as shown in Figure 3. 


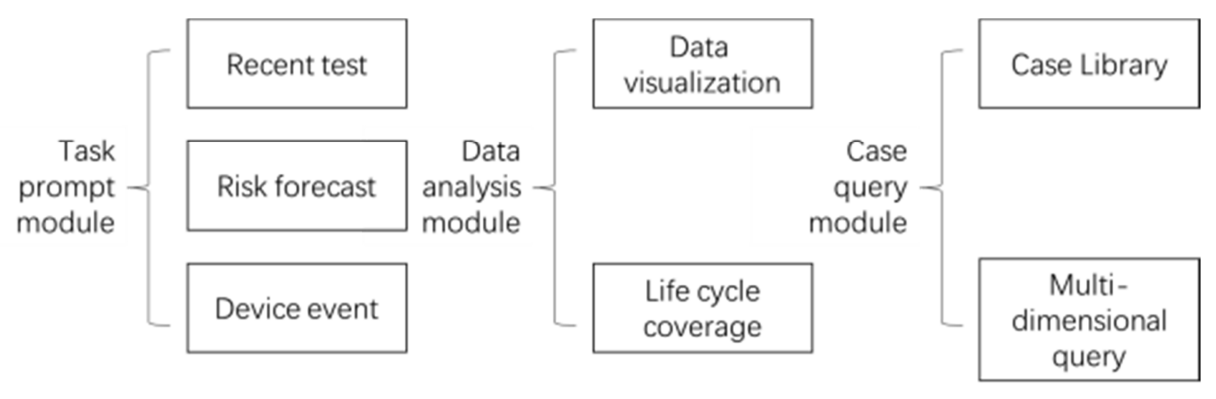

Figure 3. Functional modules of central system.

The task prompt module is responsible for recording and prompting equipment historical information. The data analysis module stores a large number of equipment data of the power utility and has a special equipment database. For existing equipment in the database, the data analysis module can diagnose the equipment using operation status data, and for equipment which is new to the database, the module can do diagnosis by referring to equipment with the same voltage level and similar type. The module can also do a statistical analysis on the occurrence times of a variety of failure and provide a basis for deducing the cause of failures. The central system contains a comprehensive fault case library, including equipment main technical parameters, fault name, fault location, fault performance, fault level, possible derivative failure, abnormal state value, diagnostic test, processing measures and so on. The library covers all types of fault and has a collection of more than 300 typical transformer fault cases. Fault case library can be expanded manually or automatically and the fault diagnosis reliability of the central side will gradually increase with the accumulation of the fault cases in the library.

\section{Application Scenario}

1. Standardized field work

Field work can be standardized using intelligent O\&M mobile terminals. Field worker will strictly follow the prompts and steps on the terminal. Besides, the quality of field operation can be ensured and evaluated objectively with video recordings of key steps of field work. Standardized operational flow is the established and interactive tips are provided to field operators with the introduction of State Grid guidelines and on-site operation requirements. These measures effectively reduce problems caused by inexperienced field personnel and improve O\&M quality.

\section{Standardized data}

The O\&M solution standardizes the data of mainstream field equipment. Unified model data conversion interfaces are provided to ensure that the output data of the same test project are unified and standardized, which improves the accuracy of the field operation data and lays the foundation for the efficient use of the data and the realization of advanced applications. It helps to improve utility's asset management capability.

3. Central side supporting field work
The field work data are transmitted to the intelligent O\&M terminal for recording, analysis and real-time evaluation of equipment status. Central side support services will be activated when O\&M personnel are confronted with complex and difficult problems These services include data call, advanced analysis, remote expert support etc. The central side support services effectively support on-site analysis and diagnosis work, help to detect potential defects and state deterioration and improve the practicality of advanced applications.

4. Customized application services

Customer tailored solutions with the same architecture and customized services can be provided according to customer's actual needs and the characteristics of customer's power grid. Customized services include threshold settings, focus, algorithm customization, report output etc. Customized services, in line with the company's differentiated models and applications, can be used and expanded by users. Power company's O\&M can be supported efficiently with customized services.

5. One-click data upload function

The one-click data upload function effectively simplifies data entry and job application process (work ticket application, data records and data entry from PMS, etc.). It also avoids possible mistakes of workflows missing and recording errors and improves work efficiency and experience. With one-click data upload function, O\&M personnel will have more time and energy dedicated to intelligent storage of field data. It contributes to knowledge transmission and sharing.

\section{Application Cases}

The integrated O\&M solution has been applied successfully in power utilities in a number of provinces and cities in the company. A data analysis center is constructed by integrating O\&M data of power utilities. Various customized services are provided. Meteorological environment data are integrated during equipment diagnosis in complex weather. Reactor state monitoring algorithm is optimized. Mobile O\&M platform is applied for live detection vehicles. Live overhaul analysis is carried out for live detection such as infrared and partial discharge. Advanced application model is completed in equipment O\&M management based on "reliability" theory. Weekly and monthly substation equipment assessment function is provided to meet the needs of multiple provinces and cities. 
Power grid equipment status assessment and diagnosis systems are launched in a number of provinces and cities. More than 5,000 times of main transformer (reactor) access and analysis have been done, generating thousands of diagnostic reports and completing more than 300 cases of diagnosis. Defects of main transformers and reactor multiple equipment have been detected by big data analysis center for several times and reasonable troubleshooting solutions are given. It helps to avoid significant loss.

\section{Conclusion}

The integrated power grid equipment O\&M solution based on big data, cloud computing, the Internet of Things and mobile Internet improves field workers' O\&M capability and effectively guarantees stable and reliable grid operation. It is of positive significance to apply the solution in power grid system.

\section{References}

[1] Zhong Lei. Application and Management of Intelligent Operation and Management Platform of Transmission Line [J]. Jiangxi Jingdezhen Power Supply Company, December 2014.

[2] Wu Hongli. Study on the Decision Model and Management Process of Transformer Equipment Maintenance in the Background of Smart Grid [J]. Southeast University, 2013.

[3] Liu Tao, Dai Shuguang. Research on the Intelligent Operation and Maintenance Platform of Transmission Line [J]. North China Electric Power University, 2012.

[4] Tang Haitao. Analysis of Power Grid Operation and Maintenance Risks and Technical Overhaul [J]. Communications World, 2014 (20).

[5] Liu Yang. Analysis of the Process of Integrated Operation and Maintenance of Transformer [J]. China High-tech Enterprise, $2013(20)$

[6] RICHARD D., CHERRY T.. Standards for the Smart Grid [C]. Proceedings of IEEE Energy 2030 Conference, November, 17-18, 2008:1-7.

[7] Yu Yixing, Ruan Wenpeng. Smart Grid [J]. Power Grid and Clean Energy Resource. 2009, 25(1):7-11.
[8] Xie Kai, Liu Yongqi, Zhu Zhizhong. The Future Smart Grid. Chinese Electric Power, 2008, 41(6): 19-22.

[9] Liu Huajun. Research on the architecture of Internet of things. Chinese new Communication. 2010(9): 17-21.

[10] Wang Dewen, Song Yaqi, Zhu Yongli. Information Platform of Smart Grid Base on Cloud Computing. Power system automation 2010, 34(22): 7-12.

\section{Biography}



Liang Dong (1969-), Male, Master, Senior engineer, long-term engagement in power system O\&M management.

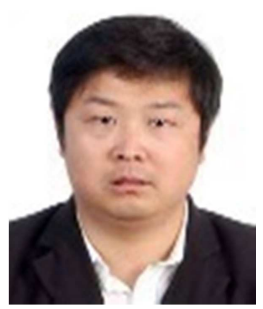

Su Yirong (1977-), communication author, Male, Master, Senior engineer, long-term engagement in research and development of smart grid technology.

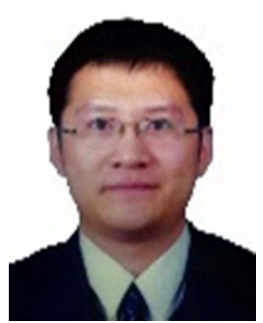

Liu Zhiwei (1981 -), Male, Master, Senior engineer, long-term engagement in research and development of smart grid technology.

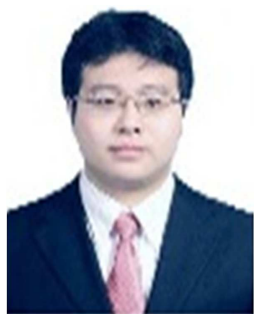

Liu Shen (1988-), Male, Master, Engineer, long-term engagement in power system O\&M management. 